\title{
Hepatitis B vaccination schedules in genitourinary medicine clinics
}

\author{
D Asboe, P Rice, A de Ruiter, J S Bingham
}

\begin{abstract}
Objectives: To compare two vaccination schedules in delivering hepatitis $\mathrm{B}$ vaccine to at-risk genitourinary medicine clinic attenders.

Setting: Genitourinary medicine clinic of St Thomas' Hospital, London, UK.

Methods: Two vaccination protocols were compared. Between January 1991 and December 1992, individuals had doses of recombinant hepatitis B vaccine at 0,1 and 6 months (standard). From January until October 1993 doses of vaccine were administered at 0, 1 and 2 months (accelerated), following which timing of a booster dose was made on the basis of hepatitis B surface antibody (anti-HBs) assessment. Case notes were reviewed with regard to compliance rates and anti-HBs levels.

Results: Two hundred and fourteen individuals were included (standard 104, accelerated 110). Of the standard group $80.8 \%$ and $61.5 \%$ attended for the 2 nd and 3rd doses respectively compared with $80.0 \%$ and $75.5 \%$ of the accelerated group (attendance for the 3rd dose $\chi^{2}=4 \cdot 19, \mathrm{p}<$ $0.05)$. For both of these groups compliance was significantly better in those who requested vaccination rather than being offered it opportunistically $\left(\chi^{2}=4.86, \mathrm{p}<0.05\right)$. Seroconversion rates were not significantly different between the two groups $(87 \cdot 5 \%$ versus $83 \cdot 1 \%)$. A significantly higher proportion of the standard group, however, achieved anti-HBs levels greater than $100 \mathrm{iu} / \mathrm{l}$. Conclusions: Completion of hepatitis $B$ vaccination was improved by changing to a 0,1 and 2 month protocol. Levels of anti-HBs achieved in the accelerated group, however, were lower. If it is confirmed that maintaining anti-HBs levels is not important in retaining protection against hepatitis B then the accelerated schedule has clear advantages. If not, the advantages may be nullified by the need, in some, for an early booster.

(Genitourin Med 1996;72:210-212)
\end{abstract}

Keywords: hepatitis B; vaccination; genitourinary medicine clinics; compliance

\section{Introduction}

The introduction of safe and effective hepatitis $B$ vaccines in the 1980 s has presented the medical profession with the opportunity to prevent an infection with serious short and long term sequelae. Because of the relatively low prevalence rates within the United Kingdom, it has been policy to vaccinate specific targeted populations ${ }^{1}$ - that is to identify groups at higher risk of acquiring infection and to vaccinate them-rather than the universal approach being used in an increasing number of countries with higher prevalence. ${ }^{2}$

Genitourinary medicine (GUM) plays an important role in this approach, as some of the higher risk groups identified for targeting, especially gay and bisexual men, access clinic services. With all vaccination programmes, however, one of the obstacles to achieving immunisation is poor individual compliance in completing the required schedule. This is emphasised in GUM because of a young and mobile (in this case inner-city) population and the sporadic nature of clinic attendance. The recommended schedule for vaccination consists of doses of vaccine at 0,1 and 6 months, with antibody titres checked 2 months following vaccination. ${ }^{1}$ However, an audit of hepatitis B vaccination in a GUM clinic setting using this schedule found that only $68 \%$ of individuals completed the course. ${ }^{3}$ This fact coupled with concern at compliance within our own clinic led us, in late 1992, to alter our pro- gramme to a 0,1 and 2 month vaccination schedule, following which a booster dose was recommended at a time determined by serology performed 1 to 2 months following the 3rd injection. It was considered that this may enhance attendance and so was incorporated into the clinic protocol.

The aim of this study was to compare and contrast the two schedules in delivering hepatitis B vaccine to GUM clinic attenders with regard to compliance and post-vaccination antibody levels.

\section{Material and methods}

We performed a retrospective casenote review on individuals attending the GUM clinic of $\mathrm{St}$ Thomas' hospital who commenced hepatitis B vaccination between January 1991 and October 1993. All individuals, were demonstrated to be hepatitis B core antibody (anti-HBc) negative (Abbott EIA), prior to vaccination.

During this time, two different vaccination schedules were used. Between January 1991 and December 1992 a 0, 1, 6 month schedule was used. From January 1993 onwards a change was made to a $0,1,2$ months schedule. All patients were asked to return 1 to 2 months after the 3rd dose for measurement of serum hepatitis B surface antibody (anti-HBs). Samples where the titre was more than $150 \mathrm{iu} / 1$ were not diluted to give a precise titre and so 
Table 1 Patient characteristics

\begin{tabular}{lcc}
\hline & Standard & Accelerated \\
\hline Number of patients & 104 & 110 \\
Mean patient age, years & $30 \cdot 1(17-64)$ & $30 \cdot 0(18-57)$ \\
Percentage male & $97 \cdot 2$ & $97 \cdot 1$ \\
Risk factor (\%) & & \\
Gay/bisexual & $91 \cdot 8$ & $91 \cdot 3$ \\
Het male & $4 \cdot 5$ & $5 \cdot 7$ \\
Het female & $2 \cdot 7$ & $2 \cdot 8$ \\
IVDU & $1 \cdot 0$ & $0 \cdot 0$ \\
\hline
\end{tabular}

are represented as $>150 \mathrm{iu} / 1$. In those vaccinated at 0,1 , and 2 months the anti-HBs results led to the following recommendations:

$\begin{array}{ll}\text { anti-HBs } & \text { recommendation } \\ <10 & \text { repeat vaccination course } \\ 10-50 & \text { booster dose immediately } \\ 51-100 & \text { booster dose } 1 \text { year } \\ 101-150 & \text { booster dose } 2-3 \text { years } \\ >150 & \text { booster dose } 5 \text { years }\end{array}$

Patients with either no response or a good level of anti-HBs after immunisation were advised regarding further booster doses in accordance with the published guidelines from the Department of Health's Joint Committee on Vaccination and Immunisation. ${ }^{1}$ Those patients with poor responses of between 10 and 50 and 51-100 iu/l were managed in general agreement with the findings of a study examining persistence of antibody in vaccinees. ${ }^{4}$

Vaccination was with yeast-derived, recombinant hepatitis B vaccine (20 ug Engerix B, SmithKline Beecham) injected intramuscularly. Neither individuals known to be HIV antibody positive, nor those indicating they would complete vaccination elsewhere were included in this analysis. Importantly, during the time covered by this study, there was no recall system in place for those failing to attend for vaccination.

\section{Results}

A total of 224 individuals were identified. Of the 214 sets of notes which were located, 104 individuals were vaccinated via the standard (std) protocol, and 110 via the accelerated (acc) schedule. Demographic and risk factor characteristics of the two groups were comparable (table 1 ).

Thirty-eight $(63.5 \%)$ of the standard group and $43(60.9 \%)$ of the accelerated group were either HIV antibody negative at the initiation of vaccination, or have subsequently tested negative. In the remainder HIV status was unknown. In 43 individuals $(20.5 \%)$ it was clear from the notes that the individual con-

Table 2 Numbers (percentage) of individuals attending each stage of vaccination programme

\begin{tabular}{llrlll}
\hline & & \multicolumn{1}{c}{ 1st inj } & 2nd inj & 3rd inj & Serology \\
\hline Standard & requested & $22(100)$ & $20(90 \cdot 0)$ & $18(81 \cdot 8) \dagger$ & $14(63 \cdot 6)$ \\
& opportunist & $82(100)$ & $64(78 \cdot 0)$ & $46(56 \cdot 1) \dagger$ & $26(31 \cdot 7)$ \\
& total & $104(100)$ & $84(80 \cdot 8)$ & $64(61 \cdot 5)^{\star}$ & $40(38 \cdot 5)$ \\
Accelerated & requested & $21(100)$ & $18(85 \cdot 7)$ & $18(85 \cdot 7) \dagger$ & $12(57 \cdot 1)$ \\
& opportunist & $89(100)$ & $70(78 \cdot 7)$ & $65(73 \cdot 0) \dagger$ & $47(52 \cdot 8)$ \\
& total & $110(100)$ & $88(80 \cdot 0)$ & $83(75 \cdot 5)^{\star}$ & $59(53 \cdot 6)$ \\
\hline
\end{tabular}

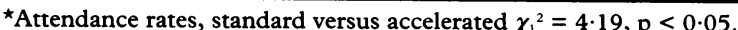

tAttendance rates, requested versus opportunistic (standard plus accelerated) $\chi_{1}{ }^{2}=4 \cdot 86$, $\mathrm{p}<0.05$.
Table 3 Number of individuals (percentage) versus level of post-vaccination hepatitis $B$ surface antibody

\begin{tabular}{lcc}
\hline Anti-HBs (iull) & $\begin{array}{l}\text { Standard } \\
(n=40)\end{array}$ & $\begin{array}{l}\text { Accelerated } \\
(n=59)\end{array}$ \\
\hline$<10$ & $5(12 \cdot 5) \dagger$ & $10(16 \cdot 9) \dagger$ \\
$10-100$ & $5(12 \cdot 5)^{\star}$ & $26(44 \cdot 0)^{\star}$ \\
$>100$ & $30(75 \cdot 0)^{\star}$ & $23(38 \cdot 9)^{\star}$ \\
\hline
\end{tabular}

* Standard versus accelerated and level of post-vaccination anti-HBs $\chi_{!}^{2}=11.58, \mathrm{p}<0.001$

HIncrease in the non responder rate amongst accelerated group $0.044 \pm 0.137$ ( $95 \%$ confidence interval). $\chi_{1}^{2}=1 \cdot 06$, $\mathrm{p}>0.5$.

cerned had requested vaccination (std $21 \cdot 1 \%$, acc $19 \cdot 1 \%$ ). It was assumed vaccination was offered opportunistically for the remainder.

The percentage of individuals attending at each stage of the vaccination programme is shown in table 2 . Included are attendance figures stratified into those either requesting or being offered vaccination. Inclusion in this analysis was determined by attendance for the 1 st dose; therefore attendance at this stage, by definition, is $100 \%$. For the 2 nd dose attendance was similar in both groups. For the 3rd dose however, attendance was significantly better in the accelerated group $\left(\chi^{2}=4 \cdot 19, \mathrm{p}<\right.$ $0 \cdot 05)$. These differences were maintained when attendance rates for post-vaccination serology assessment were examined. Also, we found that attendance was better in those who requested vaccination, with little difference tsween the standard and accelerated groups.

Thirteen patients undergoing accelerated vaccination were deemed to require an immediate booster (as a result of post-vaccination serology) of whom 10 were given this on returning for the serology result. Three did not attend. Nine out of $13(69 \cdot 2 \%)$ who were recommended a booster in 1 years time complied. Twenty-three had anti-HBs levels of greater than $100 \mathrm{iu} / 1$ and so were recommended to have a booster dose in 2 to 5 years' time. Of the 5 who had anti-HBs levels of between 10 and $100 \mathrm{iu} / 1$ following standard vaccination, 4 returned and were given a booster. Those with levels greater than $150 \mathrm{iu} / 1$ were advised to have the levels checked in 5 years.

Post-vaccination anti-HBs results are shown in table 3. There was no significant difference in the percentage of responders (with this defined as those achieving an anti-HBs level of greater than $10 \mathrm{iu} / \mathrm{l}$ ) between the two groups. There was, however, a significantly higher percentage of accelerated vaccinees achieving only a moderate response (anti-HBs between 10 and $100 \mathrm{iu} / \mathrm{l}$ ). The percentage of individuals whose HIV status was unknown and who returned for serological assessment was not significantly different in the standard and accelerated groups (std $40 \cdot 0 \%$, acc $47 \cdot 5 \%$ ).

\section{Discussion}

Immunogenecity studies of a recombinant hepatitis $B$ vaccine have shown that both the standard and accelerated schedules generate high concentrations of specific antibody. ${ }^{5}$ The findings of these studies that antibody levels at 7 months were higher under the 0,1 and 6 month schedule led to the recommendation 
that routine vaccination should follow this regimen. The studies, however, did not address the issue of individual compliance. Although Hess $^{6}$ found $99 \%$ completion rates after 3 doses (standard schedule) this was in a study of health care workers in a research setting, rather than a clinical situation. In contrast, we found completion rates using this schedule to be low. Upon changing to the 0,1 and 2 month schedule there was a significant improvement in the completion of the 3 dose schedule.

The concern that the non-parallel nature of the two groups may confound the results is valid. The rate of recruitment of the second group was faster, raising the possibility that either more (and perhaps more motivated) individuals were presenting for vaccination or that the medical staff were more effective in identifying at-risk individuals and initiating vaccination. In the latter scenario any such diligence may have translated directly into better attendance. In other aspects though the groups were similar. Firstly, individual characteristics such as age and identifiable risk factors were comparable. Secondly, for those parts of the schedule which were unchanged, completion rates were virtually identical; significant differences were observed only where the schedules diverged. If the acc group were intrinsically more compliant we might have expected improved attendance for the second injection.

The percentage of individuals who generated protective levels of antibody (anti-HBs $>10 \mathrm{iu} / \mathrm{l}$ ) was not significantly different in the two groups. Although the percentage of poor responders $(15 \%)$ was high compared with studies in healthy volunteers ${ }^{56}$ it is comparable to the $18 \%$ found by $\mathrm{Hadler}^{7}$ when vaccinating gay men with plasma derived vaccine.

A drawback of the accelerated schedule lies in the lower levels of anti-HBs that are produced. After the 3 doses only half as many recipients of the accelerated schedule achieved an antibody level of greater than $100 \mathrm{iu} / \mathrm{l}$. As the persistence of antibody is related to the peak level achieved, ${ }^{8}$ the consequence of lower levels of antibody is the shorter duration for which antibody remains above $10 \mathrm{iu} / \mathrm{l}$. It was for this reason that the recommendation was made when using an accelerated protocol that a 1 year booster be routinely given. However, rather than using this arbitrary 1 year booster, we utilised a more flexible approach. The predictability of decline in anti-HBs enables one to identify those individuals who require an early booster, whilst allowing those with higher levels a broader window during which to return. We took advantage of this by recommending a range of times for a booster dose from immediate to 5 years.

There are problems with the accelerated approach. Firstly, because there is a need to identify those requiring an early booster, attendance for post-vaccination antibody testing is important. We found, however, that only $71 \%$ of those completing the 3 dose accelerated vaccination attended at this stage. Secondly as more doses of vaccine are being used, this approach is more costly. Thirdly, for those requiring an early booster attendance for this injection is important to ensure antibody levels are maintained.

There is opinion that concern about declining levels of antibody and the subsequent risk of infection is misplaced. ${ }^{9}$ Studies have shown that although decaying vaccine-induced antibody levels render individuals susceptible to infection, (as evidenced by anticore antibody conversion) this is rarely accompanied by acute hepatitis or by the development of carrier status. ${ }^{7}$ Prevention of these sequelae is the primary goal of vaccination. Should it be confirmed that individuals in whom antibody levels declined to below $10 \mathrm{iu} / 1$ were still protected from these outcomes, the predominant concern of vaccination would be to ensure that individuals are immunised and assessed for evidence of anti-HBs conversion rather than their declining antibody levels over time. In the United States of America guidelines do not include recommendations to monitor antibody levels and boost when low. Instead reliance is made on immunological memory to maintain protection against infection when antibody levels decline below $10 \mathrm{iu} / 1 .{ }^{10}$ As individuals comply better with the accelerated regimen and there is no detrimental effect on seroconversion rates, ${ }^{56}$ the argument for this programme under these conditions would be undeniable.

If the decline of anti-HBs to below $10 \mathrm{iu} / 1$ is deemed important, however, then the advantages of the accelerated over the standard schedule are debatable. It is evident that more individuals complete the accelerated vaccination schedule but potentially at the cost of protective immunity which is not as long-lasting. Although we need to be wary about the retrospective classification of the requested and opportunistic groups it would appear there was little advantage for the group who request vaccination as their completion rates were scarcely different. For the remainder who are vaccinated opportunistically, neither the standard or accelerated protocol give highly satisfactory results. A prospective study utilising parallel vaccination groups and ideally some type of recall system would allow these findings to be confirmed or refuted.

1 Department of Health, Welsh Office, Scottish Office Home and Health Department, DHSS (Northern Ireland). Immunisation against infectious diseases. London: HM Stationery Office, 1992.

2 Kane M. Global programme for the control of hepatitis B infection. Vaccine 1995;13(Supp 1):S47-9.

3 Bhatti N, Gilson RJC, Beecham M, et al. Failure to delive hepatitis B vaccine: confessions from a genitourinary medicine clinic $B M 7$ 1991:303:97-101.

4 Ambrosch F, Frisch-Niggemeyer W, Kremsner P, et al. Persistence of vaccine-induced antibodies to hepatitis $B$ surface antigen and the need for booster vaccination in adult subjects. Postgrad Med $\mathcal{f}$ 1987;63(Suppl 2) 129-35.

5 Andre FE. Summary of safety and efficacy data on a yeastderived Hepatitis B vaccine. Am $\mathcal{f}$ Med 1989;87(supp 3A): $14 S-20 S$

6 Hess G, Hingst V, Cseke J, Bock HL, Clemens R. Influence of vaccination schedules and host factors on antibody response following Hepatitis B vaccination. Eur $\mathcal{F}$ Clin Microbiol Infect Dis 1992;11:334-40.

7 Hadler SC, Francis DP, Maynard JE, et al. Longterm immunogenicity and efficacy of hepatitis $\mathrm{B}$ vaccine in immunogenicity and efficacy of hepatitis B vaccine in

8 Tilzey AJ, Palmer SJ, Banatvala JE, Vines SK, Wilks WR. Hepatitis $\mathrm{B}$ vaccine boosting among young healthy adults. Hepatitis B vaccine boosting
Lancet 1994;344:1438-9.

9 Hall AJ. Hepatitis B vaccination: protection for how long and against what? $B M \mathcal{F} 1993 ; 307: 276-7$

10 Tilzey AJ. Hepatitis B vaccine boosting: the debate continues. Lancet 1995;345:1000-1. 